\title{
Operation Characteristics of Microwave Sources Based on Slow-Wave Interactions in Rectangular Corrugations
}

\author{
Yuki TAKAMURA, Yusuke KAZAHARI, Hiroaki OE, Kazuo OGURA, Akira SUGAWARA \\ and Mafumi HIRATA ${ }^{1)}$ \\ Graduate School of Science and Technology, Niigata University, Niigata 950-2181, Japan \\ 1) Plasma Research Center, University of Tsukuba, Tsukuba 305-8577, Japan
}

(Received 16 November 2007 / Accepted 27 March 2008)

\begin{abstract}
Studies on slow-wave devices with a novel disk cathode and two types of rectangular corrugation are reported. The beam voltage is weakly relativistic, which is less than $100 \mathrm{kV}$. A disk cathode can generate a uniformly distributed annular beam in the weakly relative region. Rectangular corrugation having the ratio of the corrugation width to the periodic length of 50 or $20 \%$ is used. Using the former, output powers of about $200 \mathrm{~kW}$ are obtained at around $100 \mathrm{kV}$. For the latter, the effect of slow cyclotron resonance is observed in the low-energy region of around $30 \mathrm{kV}$. Output powers of slow cyclotron masers are in the range of a few hundred $\mathrm{W}$. The operation mode of a slow cyclotron maser can be controlled between axisymmetric and nonaxisymmetric modes by changing the end condition of rectangular corrugation.
\end{abstract}

(C) 2008 The Japan Society of Plasma Science and Nuclear Fusion Research

Keywords: slow-wave device, weakly relativistic region, disk cathode, annular electron beam, rectangular corrugation

DOI: $10.1585 /$ pfr.3.S1078

\section{Introduction}

Microwaves of about $1 \mathrm{GHz}$ to above $100 \mathrm{GHz}$ at moderate to high-power levels (dozens of $\mathrm{kW}$ to $\mathrm{MW}$ level) are required for widespread applications such as plasma heating, plasma diagnostics, telecommunication systems and radar systems. Slow-wave high-power microwave devices such as backward wave oscillators (BWOs) are candidates for such generators. The slow-wave devices can be driven by an axially injected electron beam without the initial perpendicular velocity, and has been studied extensively as a candidate for high- or moderate-power microwave sources. In the slow-wave devices, a slow-wave structure (SWS) is used to reduce the phase velocity of the electromagnetic wave to the beam velocity. In order to increase the power handling capability and/or the operating frequency, oversized SWSs have been used successfully. The term "oversized" means that the diameter $D$ of the SWS is larger than the free-space wavelength $\lambda$ of the output electromagnetic wave by several times or more.

In Refs. [1,2], the K-band and Q-band oversized BWOs operating in the weakly relativistic region of less than $100 \mathrm{kV}$ are reported. Output power in the range of hundreds of $\mathrm{kW}$ is obtained using a sinusoidally corrugated SWS. However, their fabrications become very difficult for oversized SWSs. The sinusoidal corrugations are important in view of the discharge in the SWS by strong electric fields at an extremely high power or more of the GW. For the moderate power level of the MW or less, the discharge in the SWS may not be a serious problem. In order to over-

author's e-mail: f06e090g@mail.cc.niigata-u.ac.jp come the constructional difficulty of sinusoidal corrugation, an alternative shape of the SWS is used in this study, i.e., a rectangular shape. Rectangular corrugations can be fabricated more easily and accurately than sinusoidal corrugations. Beam interactions in a rectangular SWS become stronger than those in a sinusoidal SWS. In this study, we use two types of rectangularly corrugated SWSs for which the ratio of the corrugation width to the periodic length differs. The upper cut-off frequency is about $25 \mathrm{GHz}$ for both the SWSs. One has dispersion characteristics parallel to the sinusoidal SWS in Ref. [2], and the other has dispersion characteristics that can be newly realized using rectangular corrugation. We examine the operation characteristics of the slow-wave devices based on each SWS in the high-(around $100 \mathrm{kV}$ ) and low-beam energy region (around $30 \mathrm{kV}$ ). Moreover, we propose to use a novel disk cathode made of metal only [2]. It can generate a uniformly distributed annular beam in the weakly relativistic region.

\section{Cold Cathode}

We use a cold cathode to obtain a beam with a high current density. It is very difficult to generate a uniformly distributed annular beam using cold cathodes, especially in the weakly relativistic region of less than $100 \mathrm{kV}$. In general, hollow cathodes have been used as cold cathodes in the relativistic slow-wave devices [3]. In the past, we have also used a hollow cathode. In order to obtain an annular electron beam in the weakly relativistic region, a velvet 

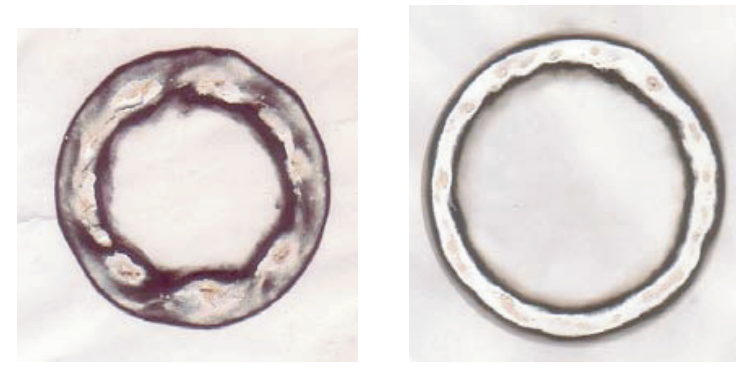

Fig. 1 The burn patterns of annular electron beam. Left-side is a pattern of 5-shot overlay for the hollow cathode with velvet at about $90 \mathrm{kV}$. Right-side is a pattern of 1-shot for the disk cathode without velvet about $80 \mathrm{kV}$.

band is stuck on the axsymmetric emitting edge [1]. The velvet functions as a dielectric fiber emitter. By adjusting the velvet, fairly uniform annular beams are obtained, as shown in Fig. 1. The beam shape is observed by the burn pattern on a thermally sensitive paper. The average radius of the annulus is nearly the same as the cathode diameter.

Recently, the uniformity of the beam was improved much more using a novel disk cathode [2]. The concept of disk cathodes was presented in Ref. [4], and was used in the relativistic region in Ref. [5]. It is easier to obtain a high-quality beam without any exacting adjustment of the shape using a disk cathode than a hollow cathode with velvet. Moreover, a disk cathode is tougher than a hollow cathode with a velvet band, and it can be produced easily and economically. In this paper, the disk cathode is tested in the weakly relativistic region. The burn pattern is shown in Fig. 1. The annular beam produced by the disk cathode is distributed more uniformly with a higher current density than the hollow cathode using the velvet. Any coating on the emission surface of cathode is not required, even in the weakly relativistic voltage less than $100 \mathrm{kV}$. In our experiments, the disk cathode operates well even around $50 \mathrm{kV}$. The average electric field between the cathode and anode is around $10^{5} / \mathrm{cm}$ or less. The beam current is some hundred amps with the current density in the range of $100 \mathrm{~A} / \mathrm{cm}^{2}$ or more.

\section{Rectangular SWS}

The cylindrical SWS is periodically corrugated, as shown in Fig. 2. Dispersion characteristics of the SWS are determined by the average radius $R_{0}$, corrugation amplitude $h$, corrugation width $d$, and periodic length $z_{0}$. The corrugation wave number is given by $k_{0}=2 \pi / z_{0}$. The dispersion characteristics of the structure are controlled by changing $R_{0}, h, d$, and $z_{0}$.

The dispersion curves of the rectangular SWS are obtained by a numerical method based on the mathematical formula in Ref. [6]. Figure 3 shows the dispersion relation of the axisymmetric transverse magnetic $\left(\mathrm{TM}_{01}\right)$ mode for type $\mathrm{A}$ and $\mathrm{B}$, whose parameters are listed in Table 1. In

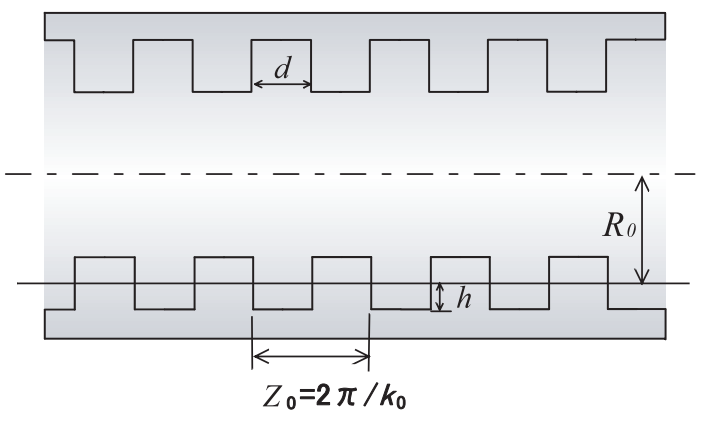

Fig. 2 Periodically corrugated cylindrical SWS.
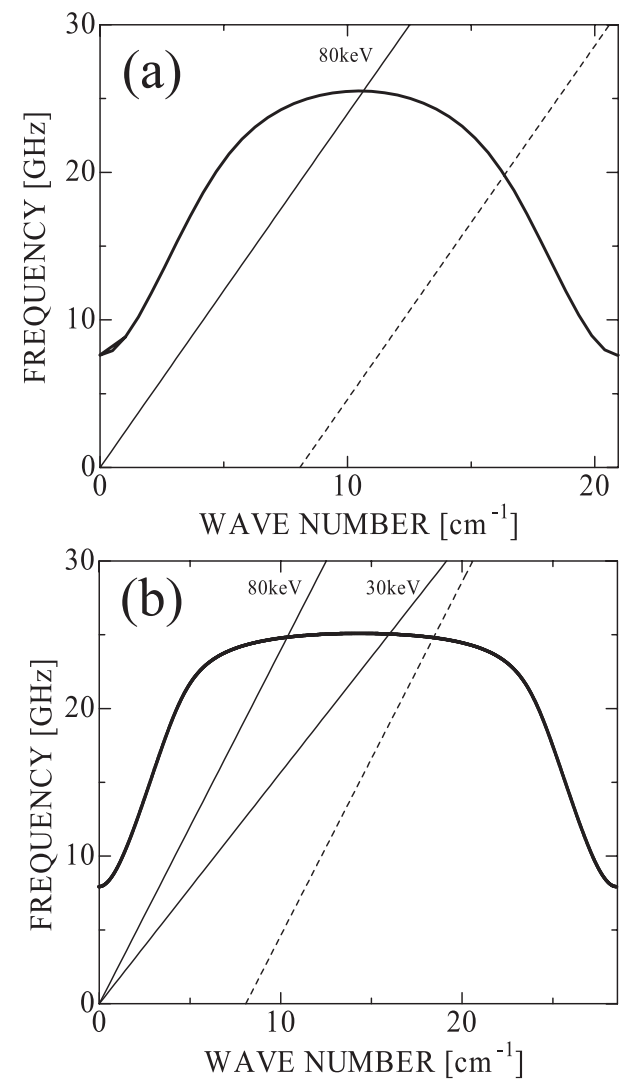

Fig. 3 Dispersion characteristics of $\mathrm{TM}_{01}$ for rectangular SWS, for type A (a) and type B (b). Solid and dashed lines are the beam line of space-charge mode and slow cyclotron mode, respectively.

Table 1 Parameters of rectangular SWS

\begin{tabular}{|c|c|c|c|c|}
\hline & $R_{0}[\mathrm{~mm}]$ & $h[\mathrm{~mm}]$ & $z_{0}[\mathrm{~mm}]$ & $d / z_{0}[\%]$ \\
\hline Type A & 15.1 & 1.1 & 3 & 50 \\
\hline Type B & 15.38 & 1.38 & 2.2 & 20 \\
\hline
\end{tabular}

Fig. 3, the beam lines of the space-charge mode $\omega=k_{z} v$ and slow cyclotron mode $\omega=k_{z} v-\Omega$ are also plotted. Here, $\omega, k_{z}, v$, and $\Omega$ are the angular frequency, wave number, beam velocity, and relativistic cyclotron frequency, respectively. The slow space charge and slow cyclotron modes couple with the fundamental $\mathrm{TM}_{01}$ mode, leading to the Cherenkov and slow cyclotron instabilities. For the 
type A SWS shown in Fig. 3 (a), the slow-wave device operates as a BWO based on the Cherenkov instability using an $80 \mathrm{keV}$ beam. The beam interaction point with $\mathrm{TM}_{01}$ is close to the upper cut-off at $\pi$ point. For the type B SWS shown in Fig. 3 (b), the dispersion curve around the upper cut-off becomes flat compared with the type A. Also, the interaction point between the $80 \mathrm{keV}$ slow space-charge mode and $\mathrm{TM}_{01}$ mode shifts toward the traveling wave region. Decreasing the beam energy to $30 \mathrm{keV}$, the interaction point moves to a backward wave region. We examine the operation characteristics of the slow-wave device using each SWS in a high (around $100 \mathrm{kV}$ ) and a low (around $30 \mathrm{kV}$ ) energy region. It should be noted that the fabrication of sinusoidal corrugation with a dispersion curve, like in Fig. 3 (b), is virtually impossible. The newly designed type B corrugation has a very flat upper cut-off region of about $25 \mathrm{GHz}$. The frequency of the Cherenkov interaction point does not decrease, even for beams around $30 \mathrm{kV}$. Our aim is to investigate weakly relativistic slow-wave devices from such a low energy to $100 \mathrm{keV}$.

\section{Experimental Results}

The experimental setup is schematically shown in Fig. 4. An output voltage of up to $100 \mathrm{kV}$ from the pulse forming line is applied to the cold cathode, which is the disk cathode proposed in Sec.2. A uniform axial magnetic field $B_{0}$ for the beam propagation is provided by ten solenoid coils. The value of $B_{0}$ can be changed from zero to about $0.9 \mathrm{~T}$. The microwave outputs are picked up by a rectangular horn antenna, typically located $600 \mathrm{~mm}$ away from the output window. Adequate amount of signal attenuation in the detecting system is provided to protect the crystal detector. Figure 5 shows an example of the detected signals for the type A SWS. The beam voltage and current are about $100 \mathrm{kV}$ and $300 \mathrm{~A}$, respectively, at the microwave peak time.

The microwave signal is split into two branches using a multi-hole directional coupler; one consists of a short waveguide and forms a prompt signal, and the other branch is a delay line and forms a delayed signal. The delay line is composed of a 31-m-long waveguide with a cutoff frequency of $17.4 \mathrm{GHz}$ and a delay time is $152 \mathrm{~ns}$. The operation frequency estimated from the delay time is about $26 \mathrm{GHz}$.

There exists a critical beam voltage for meaningful radiations [7]. The starting voltage is more critical than the starting current for the oversized BWOs. Figure 6 shows the dependence of the microwave power on the cathode voltage with type A SWS. The oscillation starting voltage is about $60 \mathrm{kV}$. The meaningful microwaves are not observed below this starting voltage. Output powers increase by increasing the cathode voltage above the starting voltage. The vertical axis in Fig. 6 corresponds to microwave power picked up by the antenna. The estimated maximum radiation power is about $200 \mathrm{~kW}$ at around $100 \mathrm{kV}$.

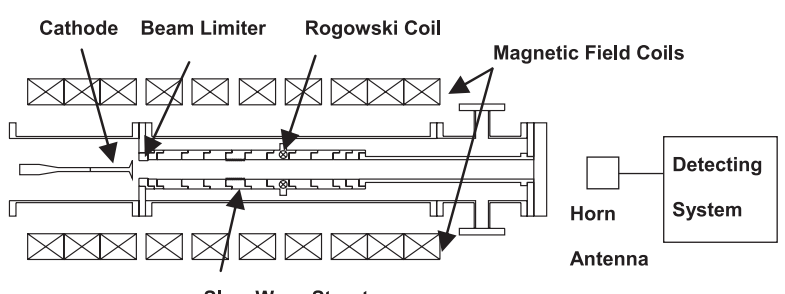

Slow Wave Structure

Fig. 4 Schematic diagram of the experimental setup.

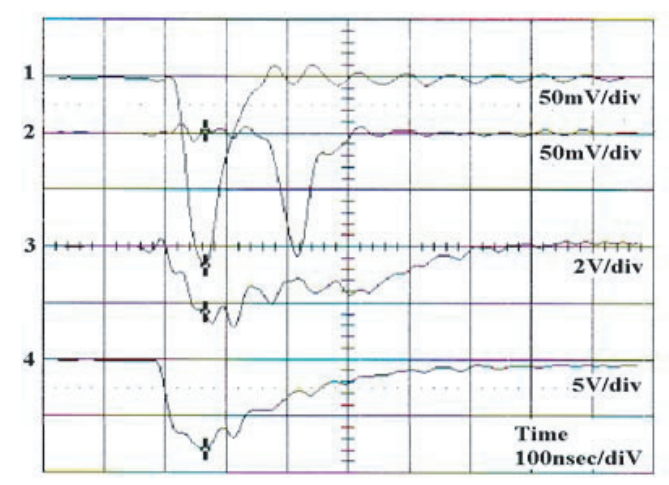

Fig. 5 Waveform of measured signals for type A SWS: 1 prompt signal, 2 delayed signal, 3 beam current signal and 4 beam voltage.

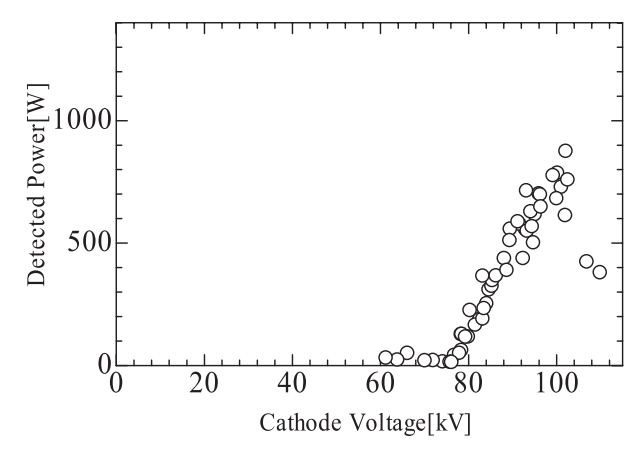

Fig. 6 Output powers versus the cathode voltage for a 10-period type A SWS.

For the type B SWS, the microwave output cannot be obtained in the energy region from 60 to $100 \mathrm{kV}$. However, microwave powers in the range of hundreds of $\mathrm{W}$ are obtained by adjusting the magnetic field $B_{0}$ in a low-voltage region around $30 \mathrm{kV}$. This voltage does not fulfill the oscillation starting condition for the type B SWS. In Fig. 7, the power dependence on $B_{0}$ for the type B SWS is shown. The microwave power picked up by the antenna is in the range of a few W. For Fig. 7, the signal attenuation factor in the detecting system is about $1 / 200$ compared with that for Fig. 6. The voltage is so low that a velvet band is required to get a good reproducibility of the beam. The microwave output power resonantly increases at about $0.65 \mathrm{~T}$, probably because of a resonance between slow cyclotron and Cherenkov interactions, as discussed later. For the type A 


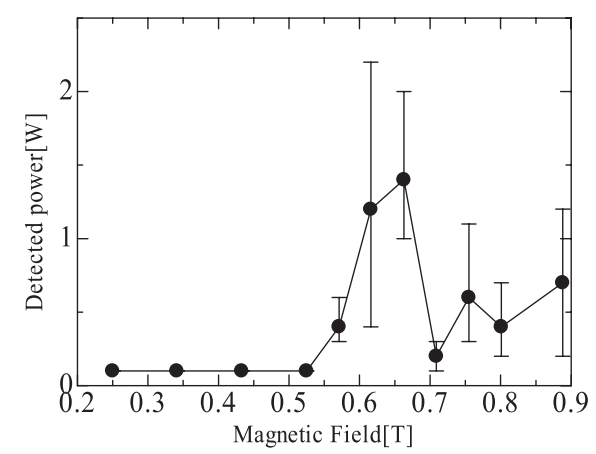

Fig. 7 Output powers versus the magnetic field for a 10-period type B SWS. The beam voltage is around $30 \mathrm{kV}$.
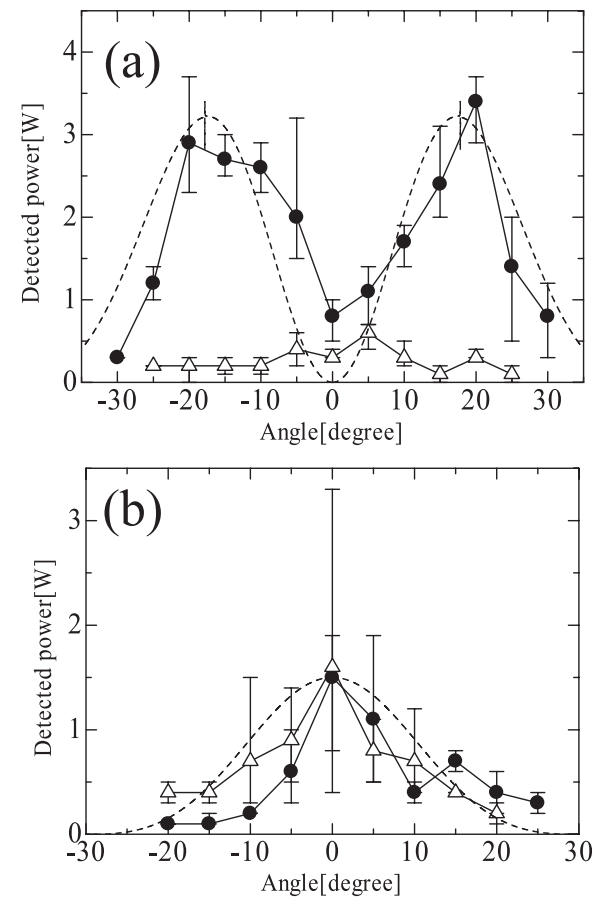

Fig. 8 Radiation patterns with the straight cylinder of (a) $34 \mathrm{~mm}$ and (b) $68 \mathrm{~mm}$ for type B SWS. $(\bullet)$ and $(\Delta)$ are respectively $E_{\theta}$ and $E_{\varphi}$ components. Dashed curves are theoretical curves of (a) $\mathrm{TM}_{01}$ and (b) $\mathrm{HE}_{11}$ mode, respectively.

SWS, such an effect of the slow cyclotron interaction has not been observed in the low-voltage region with a voltage less than the oscillation starting voltage.

For our slow-wave device, both axisymmetric $\mathrm{TM}_{01}$ mode and nonaxisymmetric hybrid $\mathrm{HE}_{11}$ mode exist as a candidate of the operation mode, as was studied based on the sinusoidally corrugated SWS case $[1,2,8]$. For determining the operating mode experimentally, the radiation patterns are measured by moving the receiving horn antenna in an equatorial plane around a pivot at the center of the output window. The electric field components of $E_{\theta}$ and $E_{\varphi}$ are measured by the horn antenna. Here, $E_{\theta}$ $\left(E_{\varphi}\right)$ is the horizontal (vertical) component of the electric field in the equatorial plane. The pure $\mathrm{TM}_{01}$ mode has no
$E_{\varphi}$ component. Also, the nonaxisymmetric hybrid $\mathrm{HE}_{11}$ mode consists predominantly of a nonaxisymmetric transverse electric $\mathrm{TE}_{11}$ component, and is characterized by a peak at the center. Figure 8 shows the radiation patterns with the type B SWS. The beam voltage is about $30 \mathrm{kV}$. The magnetic field is $0.6 \mathrm{~T}$ near the peak of the output in Fig. 7. The radiation patterns are measured by changing the straight cylinder length before the SWS in Fig. 4. For a straight cylinder of $34 \mathrm{~mm}$, the operation mode is dominated by the $\mathrm{TM}_{01}$ mode, as can be seen from Fig. 8 (a). By changing the straight length to $68 \mathrm{~mm}$, the radiation pattern changes to the pattern $\mathrm{HE}_{11}$ mode, as shown in Fig. 8 (b). The axisymmetric and nonaxisymmetric mode of the resonance operation in Fig. 7 can be controlled by the axial condition of the SWS.

\section{Discussion and Conclusion}

We propose a novel cold cathode in the weakly relativistic region, which is a disk-type cathode made of metal only. It can generate a uniformly distributed annular beam in the weakly relativistic region. We study weakly relativistic slow-wave devices with the disk cathode and two types of the rectangular SWS. The estimated output power of about $200 \mathrm{~kW}$ is obtained by using the type A SWS at about $100 \mathrm{kV}$. This is an oversized BWO operation in the weakly relativistic region. However, the microwave by the oversized BWO operation is not observed in the highenergy region of around $100 \mathrm{kV}$ for the type B SWS, because the interacting point of the beam shifts to the traveling wave region. At about $30 \mathrm{kV}$, which does not fulfill the oscillation starting condition, radiations in the range of hundreds of $\mathrm{W}$ are obtained in a certain region of the magnetic field.

The dispersion characteristic of the $\mathrm{TM}_{01}$ mode for the type B is shown in Fig. 9. Beam space-charge effects are included in the interactions using a field theory based on an infinitesimally thin annular beam in Ref. [9]. The slow cyclotron mode depends on the axial magnetic field $B_{0}$. By increasing $B_{0}$, the beam line of the slow cyclotron mode $\omega=k_{z} v-\Omega$ shifts to the right in Fig. 9. The Cherenkov interaction synchronizes resonantly with the slow cyclotron interaction at the fundamental frequency with $1.35 \mathrm{~T}$. This is a slow cyclotron maser operation, reported in Refs. [10,11]. In our experiments, resonance occurs at around $0.65 \mathrm{~T}$, which corresponds to the second harmonic of the slow cyclotron mode with $1.35 \mathrm{~T}$. The output increase in Fig. 7 can be explained by a combined resonance operation of the Cherenkov interaction and the second harmonic slow cyclotron interaction. The slow cyclotron maser operation mode can be controlled between the axisymmetric and nonaxisymmetric modes by changing the end condition of the SWS.

Although the power level at present is low, the experimental results using the type B SWS show that the synergistic coupling between the Cherenkov and slow cyclotron 


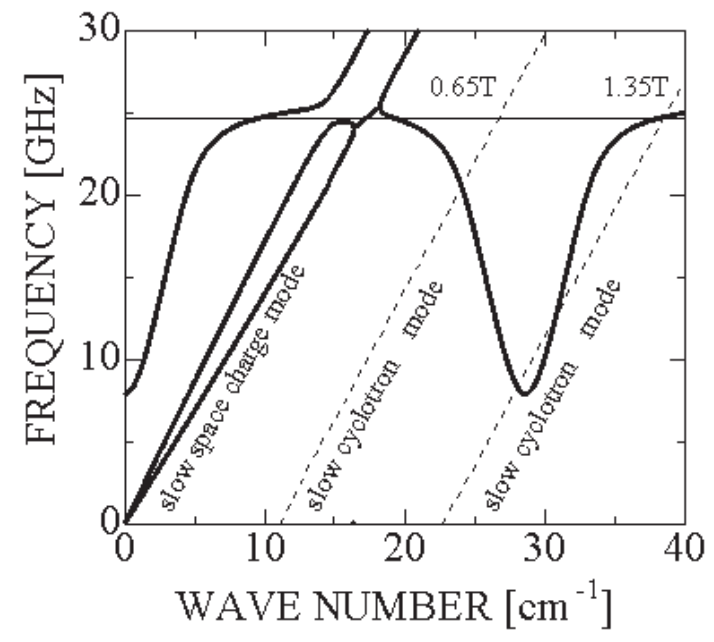

Fig. 9 Dispersion curves of fundamental $\mathrm{TM}_{01}$ for type B SWS. The beam energy is $30 \mathrm{keV}$.

interaction is effective for the second harmonic slow cyclotron interaction. The operation of the slow cyclotron maser in the low-magnetic and low-energy regions is an important result, and the maser may prove to be a candidate for a new type of useful microwave sources. More definite study of the synergistic interaction is required for developing a new type of a microwave device.

\section{Acknowledgments}

This study was partially supported by a Grant-in-Aid for Scientific Research from the Ministry of Education, Science, Sports and Culture of Japan, and was partially performed under the Collaborative Research Program between NIFS and University of Tsukuba in cooperation with Japanese universities.

[1] K. Ogura et al., IEEJ Trans. FM 125, 733(2005).

[2] S. Aoyama et al., Trans. Fusion Sci. Tech. 51, 325(2007).

[3] G.A. Mesyats, Pulsed Power (Kluwer Academic/Plenum Publishers, New York, 2005).

[4] O.T. Loza and P.E. Ivanov, Proc. 13th Int. Conf. High-Power Particle Beams, Nagaoka, Japan, pp.603-606 (2000).

[5] K. Han et al., IEEE Trans. Plasma Sci. 30, 1112 (2002).

[6] P.J. Clarricoats and A.D. Olver, Corrugated Horns for Microwave Antenna (Peter Peregrinus, London, 1984).

[7] K. Ogura et at., J. Plasma Fusion Res. SERIES 6, 703 (2004).

[8] Y. Kiuchi et al., Trans. Fusion Sci. Tech. 51, 331(2007).

[9] K. Ogura et at., J. Plasma Phys. 72, 905 (2006).

[10] K. Ogura et at., Phys. Rev. E 53, 2726 (1996).

[11] K. Ogura et at., J. Phys. Soc. Jpn. 67, 3462 (1998). 